\title{
Comparing the Accuracy of Cone Beam Computed Tomography, Digital Intraoral Radiography and Conventional Intraoral Radiography in the Measurement of Periodontal Bone Defects
}

\author{
Dehghani M', Montazer Lotf Elahi $\mathbf{H}^{2}$, Moeini $\mathbf{M}^{3}$, Bardal $\mathbf{R}^{4^{*}}$ \\ ${ }^{1}$ Assistant Professor, Radiology Dept, Yazd University of Medical Sciences. \\ ${ }^{2}$ MD. Fellowship of Pediatric Neurology, Pediatrics Dept, Tehran University of Medical Science. \\ ${ }^{3}$ Oral \& Maxillofacial Radiologist. \\ ${ }^{4}$ Assistant Professor, Radiology Dept, Qazvin University of Medical Sciences.
}

\begin{tabular}{l}
\hline ARTICLE INFO \\
\hline Article Type \\
Orginal Article \\
\hline Article History \\
Received: April 2015 \\
Accepted: August 2015 \\
ePublished: Junuary 2016 \\
Keywords:
\end{tabular}

Cone beam computed, tomography,

Dental radiography, Digital radiography, Periodontal disease, \begin{abstract}
Background and Aim: Cone beam computed tomography (CBCT) produces highquality data in periodontal diagnosis and treatment planning. The aim of this study was to compare the accuracy of CBCT with intraoral digital and conventional radiography in the measurement of periodontal bone defects.

Methods and Materials: In this diagnostic research, two hundred and eighteen artificial osseous defects (buccal and lingual infra-bony, inter proximal, horizontal, crater, dehiscence and fenestration defects) were shaped in 13 dry mandibles. CBCT and intraoral radiography with parallel technique by conventional film and digital sensor were compared with the standard reference (digital caliper). Inter and intra observer agreement were assessed using Intra class correlation co-efficient and Pearson correlation. Paired T-Test was applied for the comparison of absolute differences of conventional and digital intraoral radiography and CBCT measurements with the gold standard. All statistical analyses were performed using SPSS® v13.0 statistical software.

Results: Inter and intra observer agreement were both high for CBCT (ICC: $\alpha=0 / 88$ ) but moderate for intraoral conventional radiography (ICC: $\alpha=0 / 54$ ) and digital radiography (ICC: $\alpha=0 / 73$ ). No significant differences were detected between the observers for all the techniques $(\mathrm{P}>0.05)$. According to Paired T-test, mean difference for CBCT technique $(0.01 \mathrm{~mm})$ was lower than digital radiography $(0.47 \mathrm{~mm})$ and conventional radiography $(0.63 \mathrm{~mm})$. CBCT allowed the measurement of all lesion types, but intraoral radiography did not allow the measurement of buccal and lingual defects.

Conclusion: The results of this study showed that the studied radiographic modalities are useful in identifying the periodontal bone defects. CBCT technique showed the highest accuracy in the measurement of periodontal bone defects compared with digital and conventional intraoral radiography.
\end{abstract}

Please cite this paper as:
Dehghani M, Montazer lotf elahi H, Moeini M, Bardal R. Comparing
the Accuracy of Cone Beam Computed Tomography, Digital Intraoral
Radiography and Conventional Intraoral Radiography in the
Measurement of Periodontal Bone Defects . J Res Dentomaxillofac Sci.

*Corresponding author:

Roghieh Bardal,

Email: r2009.bardal@gmail.com,

Tel: : +98912 3825061 


\section{Introduction:}

Early detection of periodontal disease is important to prevent tooth loss. ${ }^{(1)}$ The incidence of this disease has increased ${ }^{(2)}$ and has been estimated to be about $30 \%$ in the western countries. (3) Three-dimensional (3D) observations and the ability to measure the different aspects of alveolar bone are important in the detection of periodontal disease. Previous studies have revealed that the height of alveolar bone is more useful in determining the treatment protocol and prognosis compared with the measurements from the CEJ or the depth of intra osseous lesions. ${ }^{(4)}$ Although different diagnostic techniques including probing and radiologic methods such as panoramic, bitewing and periapical radiography have been introduced, but providing a two-dimensional view is the major limitation of these methods. ${ }^{(5,6)}$

Computed tomography can overcome this limitation by providing 3D data. ${ }^{(5)}$ However, disadvantages such as higher radiation dose, greater costs and low diagnostic resolution led the scientists to implement a more accurate diagnostic modality. Nowadays, cone beam computed tomography (CBCT) is being used in many dental fields. ${ }^{(6)}$ Several studies have evaluated the accuracy of this modality. ${ }^{(1-5,7,8)}$ Although all these studies have shown the appropriate accuracy of this method, but the small sample size is their major limitation. Therefore, this study was designed to evaluate the accuracy of CBCT, intraoral digital radiography and intraoral conventional radiography in the detection of periodontal infra-bony defects.

\section{Methods and Materials:}

This diagnostic research was conducted on thirteen dry human mandibles at the Anatomy Department of Yazd and Hamedan Universities of Medical Sciences. Two hundred and eighteen defects were formed in the dry mandibles. ${ }^{(5)}$ These defects had nine different types, which included: (three-wall $(n=19)$, two-wall $(n=16)$ and onewall $(n=14)$ lesions, horizontal buccal and lingual bone lesions $(n=57)$, dehiscence $(n=15)$, fenestration $(\mathrm{n}=15)$, buccal and lingual furcation involve- ment $(n=30)$, even and uneven craters $(n=25)$, buccal and lingual infra-bony defects $(n=17)$. These defects were formed with 1 and 0.8 fissure burs and $1 / 2$ and $1 / 4$ round burs. ${ }^{(5)}$ (Figure 1 )

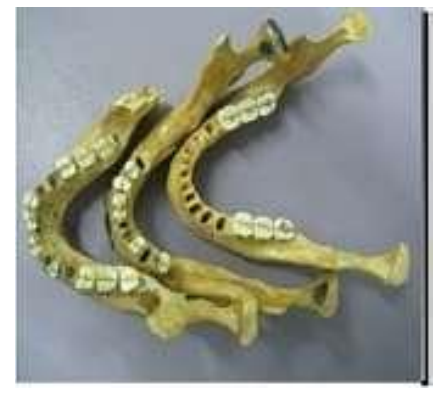

Figure 1-1- dry mandible

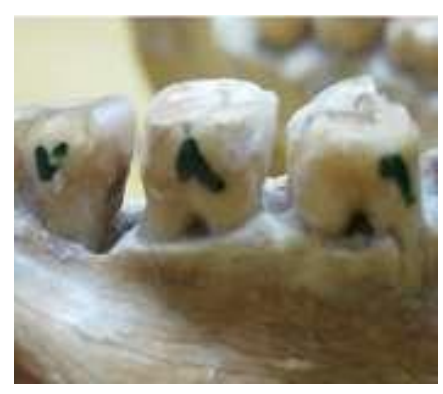

Figure 1-2- infrabony lesion

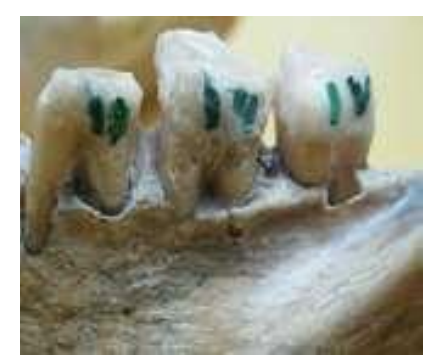

Figure 1-3- infrabony lesion

In order to signify the occlusion surface, an orthodontic wire was used as a marker. For all the lesions, the orthodontic wire was a reference point except for the horizontal lesions, which the reference point was set at the CEJ. The measurements in this study were performed with a modified digital caliper. (Figure 2) 


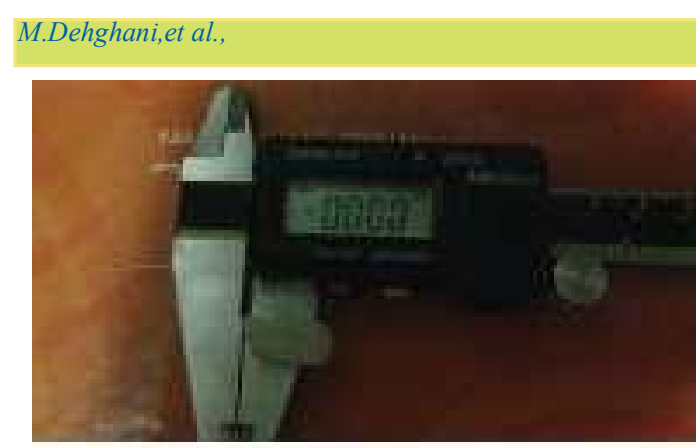

Figure 2- Digital caliper

The caliper was entered into the depth of the defects with a $0.6 \mathrm{~mm}$ orthodontic wire. For determining the horizontal defects in buccal or lingual surfaces, measurements were done in three different zones: mesio-buccal, mid-buccal and disto-buccal. The deepest level was recorded. ${ }^{(5)}$ For evaluation of bone destructions in the dehiscence of buccal, lingual and interproximal infra-bony lesions, the deepest level was recorded. In fenestrations, we measured the mesiodistal transversal length. For crater defects, the deepest space between the orthodontic wire and the bottom of the crater was measured. If the crater was uneven, the difference between buccal and lingual surfaces was also measured.

Before taking any radiographs, the mandibles were entered into a plexi glass box filled with water in order to simulate soft tissue. ${ }^{(8)}$ Images were taken with Planmeca Prostyle device (Helsinki, Finland) using long cone and parallel method with XCP film holder and E-Speed Kodak size 2 films (vee kay brothers, Panjabi, India). Focal spot-object distance was $30 \mathrm{~cm}$. Radiographic parameters were $\mathrm{KVP}=60$ and $\mathrm{mAs}=0.25$ for posterior teeth and $\mathrm{KVP}=60$ and $\mathrm{mAs}=0.20$ for anterior teeth. The radiographs were processed in an automatic processor (Hope, America) in fresh processing solutions (champion, licensed by England) in a labyrinthine darkened chamber equipped with a safety lamp (GBX-2; Kodak, Eastman Kodak Company, USA). The measurements were made on the view box with a digital caliper (Figure 3).
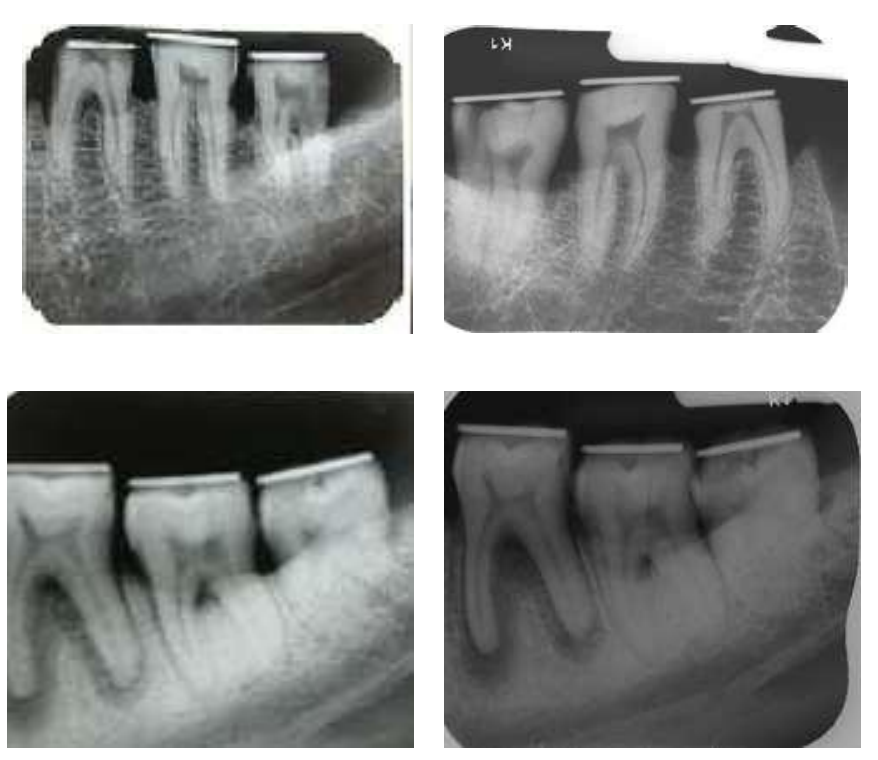

Figure 3-Intraoral radiographs of periodontal lesions obtained by kodak film (a) and PSP (b) with parallel method.

In digital radiographic method, we used PSP sensors and the intraoral radiographs were taken with ProlinX device (Planmeca, Helsinki, Finland) using long cone and parallel method with XCP film holder. Focal spot-object distance was $30 \mathrm{~cm}$. Radiographic parameters were KVP $=60$ and $\mathrm{mAs}=0.10$ for posterior teeth and $\mathrm{KVP}=60$ and $\mathrm{mAs}=0.06$ for anterior teeth. The digitalized radiographic images were transferred to the Scanora software (Soredex, Finland). After calibration of the linear distance measuring tool, the depths of the lesions were evaluated. CBCT scans were made by Promax 3D device (Planmeca, Helsinki, Finland)

(Figure 4)

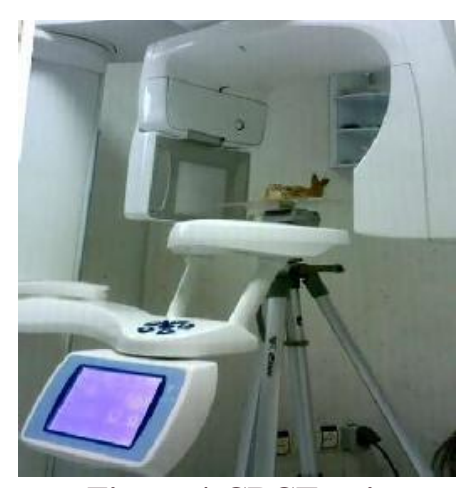

Figure 4-CBCT unit 
with the following parameters: Field of view $=88 \mathrm{~cm}, \mathrm{KVP}=70, \mathrm{~mA}=6, \mathrm{~T}=12.083 \mathrm{~s}$, Resolution $=0.125$. Each radiograph was analyzed twice by two maxillofacial radiologists in a time interval separated by one month. Data Were recorded in designated lists. After collecting the data, measurements were compared with the references using Paired T- test (SPSS ${ }^{\circledR}$ v13.0 statistical software). The validity and reliability of each technique were determined twice by a maxillofacial radiologist and a trained resident. Correlation co-efficient and Pearson correlation were evaluated and intra and inter observer agreement were measured. The values of 0.4-0.6, 0.6-0.8 and 0.8-1.0 were representative of moderate, good and high agreement, respectively.

\section{Results:}

Inter observer agreement in the detection of all the lesions was $92 \%$. In CBCT method, Pearson correlation was $87 \%$. ICC was $\alpha=0 / 73$ in Digital radiography technique while Pearson correlation was equal to $23 \%$. Conventional intraoral radiography had ICC of $\alpha=54 \%$ and Pearson correlation equivalent to $23 \%$. The results showed that a good agreement existed between CBCT technique and the golden standard in contrast to intraoral digital and conventional radiography. (Table 1) Although the accuracy of CBCT was higher than other methods, there was no statistical significant differences between different methods and the golden standard. (Table 1)

Table 1- Comparison of the pearson correlation, mean difference and discrepancy of CBCT, digital and conventional intraoral radiography

\begin{tabular}{llll}
\hline & CBCT/GS & Digital/GS & Intra oral film/GS \\
\hline $\begin{array}{l}\text { Pearson correlation } \\
(\mathrm{PV})\end{array}$ & $0>0.87(>0.05)$ & $0>0.05(>0.05)$ & $0>0.09(>0.05)$ \\
\hline Mean difference $\pm \mathrm{SD}$ & $0.01 \pm 1.39$ & $0.47 \pm 3.40$ & $0.63 \pm 3.85$ \\
\hline $\mathrm{P}_{\mathrm{V}}$ & 0.91 & 0.20 & 0.13 \\
\hline Discrepancy $0.5 \mathrm{~mm}$ & $65 \%$ & $18.8 \%$ & $18.3 \%$ \\
\hline Discrepancy 1 $\mathrm{mm}$ & $92 \%$ & $36.9 \%$ & $32 \%$ \\
\hline
\end{tabular}

With an acceptable discrepancy of 0.5 to $1 \mathrm{~mm}$, CBCT provided more reliable results than other diagnostic methods. (Table1) the measurements in CBCT were overestimated in $61.8 \%$ of the cases while in intraoral digital and conventional radiography, the measurements were underestimated in $53.6 \%$ and $61.7 \%$ of the cases. Discussion:

Previous studies have demonstrated the reliability of CBCT in implant placements, orthodontics and surgeries. Only few studies have evaluated this method in the diagnosis of periodontal diseases. Studies that have evaluated the extent of vertical alveolar bone defects have also showed that, there was a good agreement between radiographic and clinical findings. ${ }^{(9,10)}$ Probing and two-dimensional views of radiographic images provide limited information and cannot visualize the buccal and lingual defects. ${ }^{(6)} \mathrm{CBCT}$ technology with 3D views has resolved the mentioned limitations. ${ }^{(1)}$ Misch et al. stated that measurements with CBCT are as accurate as direct measurements using a periodontal probe and as reliable as intraoral radiographs in interproximal areas. ${ }^{(5)}$

Mol et al. stated that, if buccal and lingual defects were not detectable with intraoral radiography, CBCT could be considered as the best available technique. Considering the various benefits, CBCT is currently being considered as the best diagnostic modality in periodontology. ${ }^{(4)}$

Mol and Balasundaram compared image quality between $\mathrm{CBCT}$ and intraoral radiography in the evaluation of alveolar bone levels. They concluded that CBCT provided slightly better diagnostic and quantitative information on bone levels in 3D views compared with conventional radiography ${ }^{(4)}$

In our study, considering the limitations of two-dimensional views, the validity was found to be in average value. CBCT method showed a good validity which was in line with previous reports. ${ }^{(4,5,8)}$ Considering the results of Pearson Correlation, there was a good correlation between CBCT scans and the reference in the detection of bone lesions. However, a weak correlation was found between digital and conventional intraoral radiographic methods with the golden standard. Our findings supported the findings of the study by Misch et al (CBCT:0.62 and film: 0.53). ${ }^{(5)}$

Our study showed smaller standard deviation for CBCT compared with other methods. This result indicated the higher accuracy of CBCT. The mean difference between CBCT findings and the reference was $0.01 \mathrm{~mm}$ versus $0.63 \mathrm{~mm}$ for intraoral radiography.

In a study by Misch and colleague, the 
accuracy of intraoral radiography was reported higher compared to CBCT and probing. ${ }^{(5)}$ This difference can be attributed to different diagnostic methods. They suggested the CEJ as the reference point and used Gutta-percha as a depth measurement guidance. This reference point may change the results because CBCT is not efficient in detecting the CEJ. The use of Gutta-percha in in-vitro studies can mend the limitations of intraoral radiography and may lead to false accuracy of these radiographic images.

Vandenberghe and colleagues in 2007 showed the difference between the reference and CBCT and digital radiography to be $0.13-1.67 \mathrm{~mm}$ and $0.19-1.66 \mathrm{~mm}$, respectively. ${ }^{(8)}$ We found a higher accuracy for CBCT compared with their findings. This controversy can be attributed to the differences in the applied methods. Gutta-percha was used transversally in the CEJ line in buccal and lingual views. Therefore, the depth was determined as a space between a point and a line. In the present study, we used an orthodontic wire at the occlusal surface. Therefore, the depth was measured as a space between two points. Our method modulates the limitations.

Bonadkar et al in 2015 showed that there was a very high correlation of 0.988 between surgical and CBCT measurements. ${ }^{(11)}$ Numerous studies have reported that $\mathrm{CBCT}$ images can provide measurements of periodontal bone levels and defects, comparable with intraoral radiography. $(5,8)$ If a discrepancy of about 0.5 to $1 \mathrm{~mm}$ is acceptable, the accuracy of CBCT scans would be $65 \%$ and $92 \%$ for 0.5 and $1 \mathrm{~mm}$, respectively. Our findings supported the findings of Vandenberghe and colleagues. ${ }^{(8)}$

Leung et al. reported that CBCT measurements were not as accurate as direct measurements on the skulls but a certain discrepancy between the direct measurements and the estimated measurements of CBCT has to be considered as clinically acceptable. ${ }^{(1,12)}$

Furthermore, the diagnostic ability of CBCT has improved with the development of advanced equipment and software. A recent study showed that an improved quantification of periodontal bone defects has been achieved based on CBCT datasets using a new software. ${ }^{(13)}$

The depth of infra-bony lesions in inter proximal surfaces was determined more accurately in CBCT scans compared to the intraoral radi- ography. The standard deviations of CBCT and intraoral radiography were determined to be 0.13 and 0.26 , respectively. The high accuracy of CBCT scan was due to its ability to produce panoramic views from different sections of teeth; for example, buccal, lingual, mid buccal and mid lingual aspects and cross sectional slices. However, in two-dimensional radiographs, the depth was measured only in one view.

Our finding was in agreement with the study by Mengel and colleagues. ${ }^{(7)}$ In their study, intraoral and panoramic radiography could only visualize mesio-distal lesions and had higher standard deviations than CBCT. 3D images are ideal for evaluating the infra-bony defects and assessing the treatment outcomes. ${ }^{(14)}$

In horizontal lesions, intraoral radiography could not distinguish between the buccal and lingual surfaces. However, the bone lesion was measured with the aid of different bone densities in buccal and lingual cortical plates. In horizontal lesions, the CEJ can be useful for determining the bone lesion.

Intraoral radiographic methods had a higher spatial resolution versus CBCT and could more accurately signify the CEJ. Our findings support the findings of Mol and colleague. ${ }^{(4)}$

CBCT was more accurate than intraoral digital and conventional radiographs in the detection of crater lesions. Vandenberghe et al. concluded that intraoral radiography was significantly better in contrast, in the depiction of bone quality and delineation of lamina dura, but CBCT was superior in diagnosing crater defects and furcation involvements. (1) Digital and conventional radiographs failed to detect dehiscence and infra-bony lesions. CBCT could measure vertical lesions more accurately than horizontal lesions. This finding was not in agreement with the study by Bonadkar et al. ${ }^{(11)}$

\section{Conclusion:}

All the studied radiographic modalities are useful in identifying the periodontal bone defects. CBCT technique showed the highest accuracy in the measurement of periodontal bone defects compared with digital and conventional intraoral radiography. The decision regarding the application of CBCT in periodontology should be made after careful consideration of its benefits, 
limitations, and risks.

\section{Acknowledgments:}

The authors would like to thank Dental research center of Tehran university and The AFM center of Iran university of science and Technology for the laboratory work.

\section{Conflict of interests}

Authors report no conflict of interest related to this study.

\section{References:}

1.Vandenberghe B, Jacobs R, Yang J. Detection of periodontal bone loss using digital intraoral and cone beam computed tomography images: an in vitro assessment of bony and/or infra bony defects. Dentomaxillofac Radiol 2008;37(5):252-60.

2.Ozmeric N, Kostioutchenko I, Hägler G, Frentzen M, Jervøe-Storm PM.Cone beam computed tomography in assessment of periodontal ligament space: in vitro study on artificial tooth model.Clin Oral Investig 2008;12(3):233-9.

3.Noujeim M, Prihoda T, Langlais R, Nummikoski P. Evaluation of high resolution cone beam computed tomography in detection of simulated interradicular bone lesionDentomaxillofacRadiol 2009;38(3):156-62.

4.Mol A, Balasundaram A. In vitro cone beam computed tomography imaging of periodontal bone.DentomaxillofacRadiol 2008;37(6):319-24.

5.Misch KA, Yi ES, SarmentDP.Accuracy of Cone beam computed tomography for periodontal defect measurements. J periodontal 2006;77(7):1261-6.

6.Mischkowski RA, Scherer P, Ritter L, Neugebauer J, Keeve E, ZöllerJE.Diagnostic quality of multiplanar reformation obtained with a newly developed cone beam device for maxillofacial imaging.DentmaxillofacRadiol2008;37(1):1-9.

7.Mengel R, Candir M, Shiratori K, Flores-de-Jacoby L.Digital volume tomography in the diagnosis of periodontal defects.an in vitro study on native pig and human mandible. J Periodontal 2005;76(5):665-73.

8. Vandenberghe B, Jacobs R, Yang J. Diagnostic validity of 2D CCD versus 3D CBCT- images for assessing periodontal break down. Oral Surge Oral Med Oral pathol Oral radiolEndod2007;104(3):395-401.

9.Bolin A, Lavstedt S, Frithiof L, Henrikson C O. Proximal alveolar bone loss in a longitudinal radiographic investigation. IV. Smoking and some other factors influencing the progress in individuals with at least 20 remaining teeth.Acta Odontol Scand 1986;44(5):263-9. 10. Persson R E, Hollender L G, PerssonGR. Assessment of alveolar bone levels from intraoral radiographs in subjects between ages 15 and 94 years seeking dental care. J ClinPeriodontol 1998;25(8):647-54.

11.Banodkar A B, Gaikwad R P, Gunjikar T U, Lobo $\mathrm{T}$ A. Evaluation of accuracy of cone beam computed tomography for measurement of periodontal defects: A clinical study. J Indian Soc Periodontol 2015;19(3):285-9.

12-Leung CC, Palomo L, Griffith R, Hans M G. Accuracy and reliability of cone-beam computed tomography for measuring alveolar bone height and detecting bony dehiscences and fenestrations. Am J Orthod Dentofacial Orthop 2010;137(4):109-19.

13. Fleiner J, Hannig C, Schulze D, Stricker A, Jacobs R. Digital method for quantification of circumferential periodontal bone level using cone beam CT.Clin Oral Investig2013;17(2):389-96.

14.AlJehani Y A. Diagnostic Applications of ConeBeam CT for Periodontal Diseases. Int J Dent 2014; 2014:1-5. 